\title{
Electron Coherence and Interference in Disordered Conductors
}

Most transport phenomena in condensed matter are described by the Boltzmann kinetic equation and are classical in nature. However, there are many quantum effects in solid-state physics which involve the interference of electron waves. We once believed that strong elastic scattering destroyed all coherence and interference effects.

It became clear with the famous work of Mott and Anderson that quantum phenomena not only exist in disordered systems, but can be enhanced by disorder. The simplest and most famous example of such behaviour is the localization of electronic states in one-dimensional conductors. The states are localized due to interference between the incoming and backscattering waves; the amplitude of the wave function decays with distance $x$ as $\exp (-x / \xi)$, where the localization length $\xi$ depends on the intensity of backscattering.

Abrahams et al.'s formulation in 1979 of the one-parameter scaling hypothesis gave a solid basis for the new understanding of electron localization in conductors.

\section{Weak Localization}

In the classical picture of transport phenomena, the total probability for a particle to transfer from point $P$ near one lead to point $Q$ near a second lead (Fig. 1) is the sum of the probabilities for such transfer over all possible trajectories. In the quantum description, this result corresponds to neglecting the interference of scattered electron waves propagating along different paths. These waves have random phases under the quasi-classical condition $\lambda \ll<$, where $\lambda$ is the electron wave length and $/$ the electron mean-free path. However, there is a specific class of paths, namely, self-crossing paths (path 2 in Fig. 1) for which two waves propagating along such trajectories in two opposite directions (conjugated waves) accumulate the same phase difference. Denoting $A_{1,2}$ as the amplitudes of the probability of coming to point $\mathrm{O}$ by either clockwise or counterclockwise motion, the contribution of these paths to the probability of arriving at point $\mathrm{O}$ on the self-crossing paths (see Fig. 1) is given by

Akardi Aronov is a Head of Department at the A.F. loffe Physicotechnical Institute, St. Petersburg, 194021, Russia, and is a Corresponding Member of the Russian Academy of Sciences. $\mathrm{He}$ is presently visiting the Weizmann Institute, Rehovot. He studied at the Institute of Electrotechnical Engineering, St. Petersburg, and received a Ph.D. from the Institute of Semiconductors and his Habilitation in 1977 from the St. Petersburg Nuclear Physics Institute, where he worked from 1974 to 1990 after spending two years at the loffe Institute.

\author{
A.G. Aronov \\ Weizmann Institute of Science, Rehovot, Israel
}

The 1993 Hewlett-Packard Europhysics Prize for outstanding achievements in condensed-matter physics was awarded to B.L. Altshuler, A.G. Aronov, D.E. Khmelnitskii, A.L. Larkin, and B.Z. Spivak for pioneering theoretical work on coherent phenomena in disordered conductors.

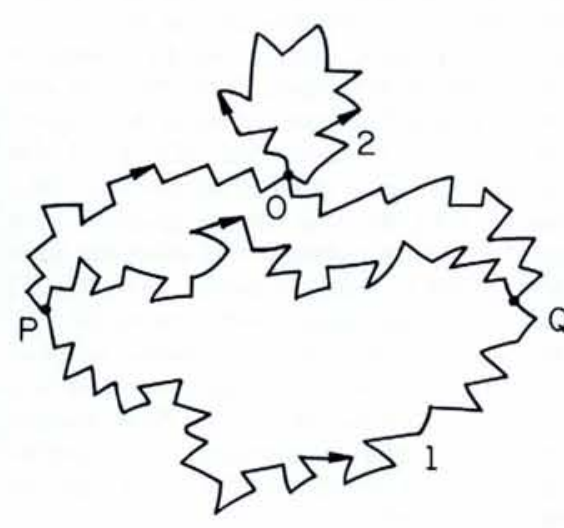

Fig. 1 - Different types of quasiclassical trajectories connecting points $P$ and $Q$. Point $O$ is the self-crossing point of a self-crossing trajectory.

$\left|A_{1}+A_{2}\right|^{2}=\left|A_{1}\right|^{2}+\left|A_{2}\right|^{2}+2 R e A_{1} A_{2}^{*}=4\left|A_{1}\right|^{2}$ which is twice the sum of the amplitude modulus squared. A higher probability of returning to point $\mathrm{O}$ means a lower probability of transfer from point $P$ to point $Q$. The interference of conjugated waves therefore favours localization of the particle, and hence results in an increase of the electrical resistance. Localization arises because the ability of electrons to diffuse through a sample is reduced and the momentum relaxation enhanced. The phenomenon was called "weak localization" (Kuz'ma Prudkov said in 1875 that "it is not surprising that scientists discovered stars and planets, but it is very surprising how they knew the names of all stars and planets").

\section{The effect of inelastic scattering}

Elastic scattering does not destroy the quantum coherence of wave functions because the Schrödinger equation in a random static potential has stationary states with exact energy levels. But the spectrum is very complicated, and leads to an anomalous frequency dependence of the conductance [Gorkov, Khmelnitskii \& Larkin, 1979]. The breaking of the wave functions' phase memory requires a change of either the energy state due to inelastic scattering, or the spin state due to spin-spin scattering on magnetic impurities. The inelastic scattering time $\tau_{\varphi}$ increases on decreasing the temperature, and it can become larger than not only the elastic time but also the time an electron takes to diffuse through a sample.

\section{Effect of a magnetic field}

If a sample is placed in a magnetic field $H$, the amplitudes of the probabilities of completing the loop on trajectory 2 of Fig. 1 in clockwise and counterclockwise directions acquire additional phase factors

$$
A_{1,2}=A \exp \left( \pm i 2 \pi \phi / \phi_{0}\right)
$$

where the magnetic flux quantum is $\phi_{0}=$ $2 \pi \hbar c / e$, the magnetic flux through the loop is $\phi=H S$, and $S$ is the projection of the area of the loop on the plane perpendicular to the magnetic field direction. It means that the phase difference $\Delta \phi$ between two conjugated waves is $2 \pi\left(2 \phi / \phi_{0}\right)$. The magnetic field therefore destroys the interference by generating a phase difference depending on the size of trajectory which, in turn, reduces the probability for a particle to return to a given point, hence reducing the resistance. This is the mechanism that accounts for the phenomenon of negative magnetoresistance [Altshuler, Khmelnitskii, Larkin \& Lee, 1980; Kawabata, 1980] - an old puzzle in metal physics discovered in tellurium by Chenzov in 1948 that remained unexplained until 1980.

The characteristic time $t_{H}$ in which the interference of conjugated waves is destroyed in a magnetic field can be estimated from the condition that the phase difference between the conjugated waves in this time becomes about unity. Owing to the diffusive character of electron motion, the area of the loop $S$ is equal to $D t$ where $D$ is the diffusion coefficient of electrons. Hence,

$$
\Delta \phi=2 H D t_{H} / \phi_{0} \approx 1 \text { or } t_{H} \approx \phi_{0} / 2 H D \text {. }
$$

\section{HEWLETT-PACKARD EUROPHYSICS PRIZE}

The Hewlett-Packard Europhysics Prize of EPS is awarded annually for outstanding achievements in condensed-matter physics. Nominations for the 1994 prize should be sent as soon as possible to the Selection Committee, H.-P. Europhysics Prize, EPS, POB 69, CH-1213 Petit-Lancy 2. 
The strength of the magnetic field which affects the conductivity is determined by the requirement that $t_{H}$ is about equal to the inelastic scattering time (i.e., $t_{H} \approx \tau_{\varphi}$ ); it is less than the classical value of the magnetic field corresponding to the curvature of the electron trajectory between two consecutive collisions with impurities.

Anomalous magnetoresistance has been investigated very carefully and good agreement with theory is obtained in all cases. Moreover, it offers a powerful method for investigating different mechanisms of phase breaking. For example, the anomalous magnetoresistance of disordered conductors may be considered as an effect of a magnetic flux brought about by confinement of the magnetic field within electron loop trajectories. The recognition that these trajectories are actually paths in real space, which can be of macroscopic size, has led to ideas concerning: a) the effect of topological differences in the shape of samples on the magnetoresistance; and b) the possibility of observing interference oscillations in the resistance of several multiply connected samples as a function of the enclosed magnetic flux [Altshuler, Aronov \& Spivak, 1981].

Consider, for example, a hollow, thinwalled, metal cylinder of radius $R$ where the perimeter of the cross-section is less than, or of the order of, $L_{\varphi}=\sqrt{D \tau_{\varphi}}$. If a magnetic field with a flux $\phi$ is applied inside the cylinder, the vector potential of the magnetic field is non-zero, its tangential component being equal to $\phi / 2 \pi R$ in the cylinder walls in which there may be no field. The presence of this vector potential gives rise to a phase difference for conjugate waves equal to $2 \pi \phi / \phi_{0}$ which is identical for all waves that return to the initial point $O$ from different sides. Each time the magnetic flux inside the cylinder is a multiple of the quantum of magnetic flux $\phi_{0}$, and hence each time the phase difference between conjugate waves is a multiple of $2 \pi$, the interference of these waves increases the probability of finding an electron at the starting point, and thus decreases the conductance of the cylinder.

Such an experiment was carried out by Sharvin and Sharvin in 1981, initially with cylindrical magnesium films (Fig. 2a), and one year later with cylindrical lithium films (Fig. 2b). Measurements demonstrated that the oscillation period of the sample's resistance $\phi_{0} / 2$ did indeed correspond to multiples of the magnetic flux through the crosssection. However, the phase of the oscillations (the derivative of the resistance at $H=$ 0 ) and the monotonic part of the resistance on increasing the magnetic field were of opposite signs for the two metals. This is a result of spin-orbit interactions [Hikami, Larkin \& Nagaoka, 1980]: in the presence of spin-orbit interaction, the character of the interference is changed, becoming destructive instead of constructive for conjugate waves with opposite spins, and the sign of magnetoresistance is changed. Lithium is a very light metal and the spin-orbit scattering is very small; in magnesium, the spin-orbit interaction is strong so the magnetoresistance and the phase of the oscillations have opposite signs to those for lithium.

The theory of weak localization predicted many other effects which have been obser-

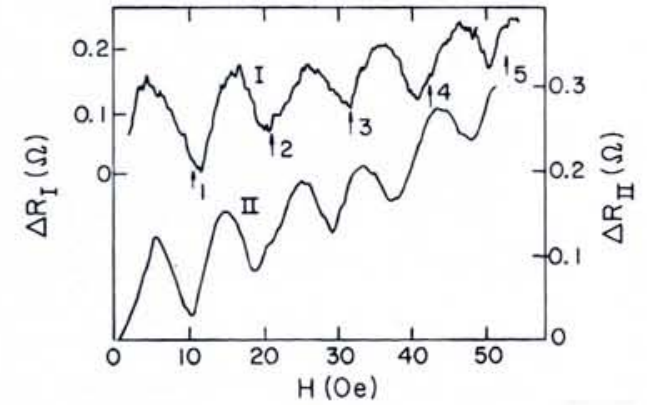

Fig. 2 - The conductivity of a long, thin-walled cylinder made of a normal metal is an oscillatory function of the magnetic flux which passes through the cylinder (the Bohm-Aharonov effect). a, left) The resistance of thin-film $M g$ cylinders (resistance $R_{l}=$

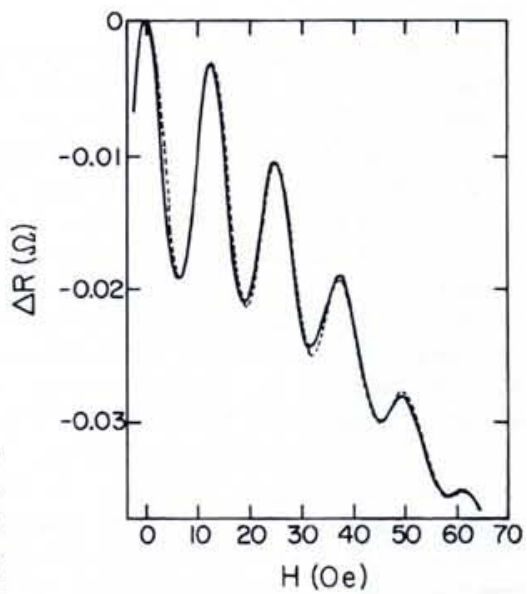

$9.2 \mathrm{k} \Omega$ and $R_{\|}=12.3 \mathrm{k} \Omega$ ), of radius $0.8 \mu \mathrm{m}$ and length $\approx 2 \mu \mathrm{m}$, as a function of the magnetic field at $1.1 \mathrm{~K}$. The arrows indicate the values of $H$ corresponding to the condition $\phi=N \phi_{0}$, where $N$ is an integer. [After Sharvin \& Sharvin, 1982]. b, right) Plots of the longitudinal magnetoresistance $\Delta R(H)$ at $1.1 \mathrm{~K}$ for a cylindrical lithium film evaporated onto a $10 \mathrm{~mm}$ long quartz filament show the excellent agreement between theory and experiment. $R(4.2 \mathrm{~K})=2 \mathrm{k} \Omega$, $R(300 \mathrm{~K}) / R(4.2 \mathrm{~K})=2.8$. Solid line: average of four experimental curves; dashed line: calculated for $L_{\varphi}=2.2 \mu \mathrm{m}$ with a $1.31 \mu \mathrm{m}$ diameter filament and a film of $127 \mathrm{~nm}$ in thickness. The filament diameter measured with a scanning electron microscope was $1.3 \pm 0.03 \mu \mathrm{m}$. [Altshuler et al., 1982; Sharvin, 1984].

ved experimentally, the most important being the temperature and size dependence of the resistance of metallic samples.

\section{Electron-Electron Interaction}

The interaction between electrons in metals is generally not weak (the potential energy of the interaction is of the same order of magnitude as the kinetic energy of the electron). On the other hand, electrons in metals can be described quite well by the free-electron approximation because the interaction between quasiparticles is weak (this is the main result of Landau's theory for a Fermi liquid). The strong interaction between electrons only produces a renormalization of parameters (effective masses, etc.) which characterize the properties of quasiparticles. For example, the density of states depends on the energy only on a scale of the order of the Fermi energy, and has no singularities near the Fermi level (i.e., electron-electron interaction leaves the density of states as a smooth function of the energy).

\section{Renormalization}

The Landau theory is based on the spatial homogeneity of the system. Impurities and defects disturb this homogeneity, so excitations with energies less than $h / \tau$, where $\tau$ is the elastic mean-free time, differ in disordered systems from those in a Fermi liquid [Altshuler \& Aronov, 1979]. This effect is also connected to quantum interference and the diffusive motion of electrons.

Consider two interacting electrons with energies $\varepsilon_{1}$ and $\varepsilon_{2}$. Let the energies be simi$\operatorname{lar}\left(\varepsilon_{1} \approx \varepsilon_{2}=\varepsilon\right)$ and the interaction time be of the order of $\hbar / \varepsilon$. In disordered metals, electrons undergo diffusive motion so there is a finite probability that electrons encounter each other again during the time interval $\hbar / \varepsilon$ (Fig. 3). All of the interaction processes occurring during the time $\hbar / \varepsilon$ will interfere, i.e., their amplitudes and not their probabilities are summed. This results in an effec- tive electron-electron coupling constant which is larger than the coupling constant without interference by a factor $1+\alpha(\varepsilon)$, where $\alpha(\varepsilon)$ is the probability that two particles encounter each other again. In a twodimensional case, for example, we have $\alpha(\varepsilon) \approx(\lambda / I) \ln (\hbar / \varepsilon \tau)$.

As in Fermi-liquid theory, this renormalized interaction changes all the physical properties of the metal, including the singleparticle density of states, conductivity, etc. The function $\alpha(\varepsilon)$ is not an analytical functities cannot be given as analytical functions of the energy, and hence of the temperature, applied voltage, magnetic field, etc.

The singularity in the single-particle density of states accounts for a well-known phenomenon called the zero-bias tunnelling current anomaly, where there is observed to be a minumum conductance as function of the applied voltage $V$ of junctions of two normal metals separated by an insulator. This conductance is proportional to the density of states of electrons of energy $\varepsilon=e V$ and is therefore not analytically dependent on $V$. Diffusion, and in particular the probability of two diffusing electrons encountering each other, depend on the dimensionality of the sample, so the renormalization factor $\alpha(\varepsilon)$ and all physical properties depend on the effective dimensionality.

Another example is a minimum in the temperature dependence of resistivity due to the renormalization of the electron-electron interaction.

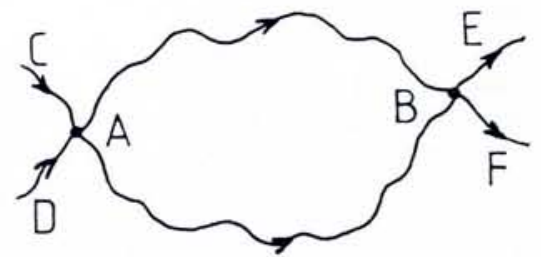

Fig. 3 - The trajectories of two particles which interact at points $A$ and $B$. The arrows indicate the direction of motion. tion of the energy $\varepsilon$ so these physical quan- 


\section{Phase relaxation}

At low temperatures, electron-electron scattering is the dominant mechanism determining the phase relaxation time $\tau_{\varphi}$ which, as has been shown above, plays a crucial rôle in weak-localization effects. The concept of a phase relaxation time is also very important in justifying the quasiparticle picture for disordered metals [Altshuler, Aronov \& Khmelnitskii, 1982], and theoretical predictions for the effect of electron-electron interactions on thermodynamic and transport properties in these materials have been confirmed by many experiments.

Owing to inelastic collisions, a wave packet acquires in a time $t$ an additional width $\Delta \varepsilon(t)$ which increases with $t$. There is a quantum mechanical uncertainty of the energy width of $\hbar / t$ and the total width of the packet is of the order of $\Delta=\hbar / t+\Delta \varepsilon(t)$. The packet has the minimum width $\Delta_{\min }$ in a time $\tau_{\varphi}$ where $\Delta \varepsilon\left(\tau_{\varphi}\right) \approx \hbar / \tau_{\varphi}$. In other words, $\tau_{\varphi}$ is the time at which the electron wave function acquires a phase $\Delta \varepsilon\left(\tau_{\varphi}\right) \tau_{\varphi} / h \approx \pi$ owing to inelastic collisions. This definition of $\tau_{p}$ is the same as the one given above. The condition for the quasiparticle description of excitations in metals to be valid is therefore given by $\Delta_{\min }=\hbar / \tau_{\varphi} \ll \varepsilon$.

The phase relaxation time $\tau_{\varphi}$ is the shortest relevant relaxation time for inelastic processes. The energy relaxation time $\tau_{\varepsilon}$ is of the same order as $\tau_{\varphi}$ if the transfer energy $\omega$ is of the order of the electron energy $\varepsilon$. However, in the case of electronelectron scattering in low-dimensional disordered systems, the situation regarding transport properties is very different compared with normal metals. This difference is a result of the small transfer energy in electron-electron collisions $(\omega<<\varepsilon)$ in disordered systems. One can show that when $\tau_{\varphi} \ll \tau_{\varepsilon}$, the energy dependence of $\tau_{\rho}$ and $\tau_{\varepsilon}$ is different (it should be underlined that in this case, the transfer energy $\omega$ can be of the same order, or less than, the state width $\hbar / \tau_{\varphi}$.)

\section{Mesoscopic Effects}

Studies of the magnetoresistance of a metal wire at low temperatures have shown that the magnetoresistance contains noiselike, but reproducible fluctuations [Webb, Washburn, Umbach \& Laibowitz, 1984] (Fig. 4). This was the first observation of a phenomenon which was later named "universal conductance fluctuations". Theory [Altshuler, 1985; Stone, 1985; Lee \& Stone, 1985; Altshuler \& Khmelnitskii, 1985] demonstrated that this effect also results from quantum interference. If the sample has a size $L=L_{\varphi}<\sqrt{D \tau_{\varphi}}$, the electron wave function conserves its coherence during the time it takes for the propagation of an electron from one lead to another.

On making measurements at length scales of order $L_{\varphi}$, the ensemble averaging which destroys the interference term in the conductance is not operable and all the interference terms contribute randomly to the resistance. Transport properties in such samples are not, therefore, self-averaging and the probability distribution of the conductance is not Gaussian with a width proportional to $N^{-1 / 2}$, where $N$ is the number of

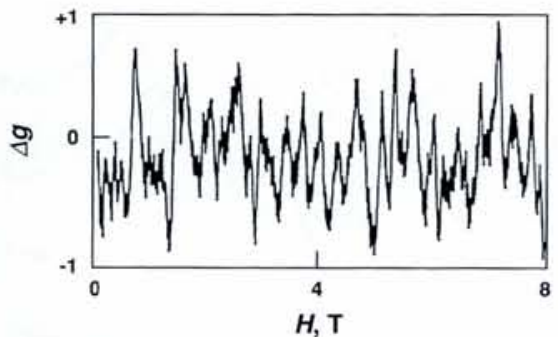

Fig. 4 - Reproducible random fluctuations in the magnetoresistance of an $\mathrm{Au}$ wire. a, upper) The resistance as a function of the applied magnetic field; $b$, lower) the autocorrelation function of the data illustrating the correlation field scale $\left(B_{c}\right.$ is the characteristic magnetic field). [From Webb et al., 1984].

size, and only weakly dependent on geometry. The heart of the universality in $\Delta g$ is the correlation of energy levels [Altshuler \& Shklovskii, 1986] - the levels must repel each other in exactly the same way as in nuclei. The increase of fluctuations on increasing the effective number of paths in the increasing volume is compensated for by a change in the rigidity of the energy spectrum

At $0 \mathrm{~K}$, all samples are mesoscopic simply because the phase memory is only destroyed by temperature or inelastic processes. The Anderson metal-insulator transition takes place at $\langle g\rangle \approx e^{2 / \hbar}$. However, the universal conductance fluctuations are of the same order of magnitude so the critical point of the Anderson transition is not well determined (the transition is not equivalent to a thermodynamic transition).

All transport effects have mesoscopic fluctuations and their relative magnitudes are of the same order as the relative magnitude of universal conductance fluctuations. Exceptions occur when the average value of the effect is zero or very small due to symmetry, since the main property of mesoscopic samples is the absence of any symmetry. The spatial symmetry is broken owing to an arbitrary distribution of impurities so it is therefore possible to observe any phenomenon if it is not forbidden by the law of the conservation of energy [Althsuler \& Khmelnitskii, 1985]. Such phenomena include, for example, the generation of even property and the result is independent average conductance and the specimen

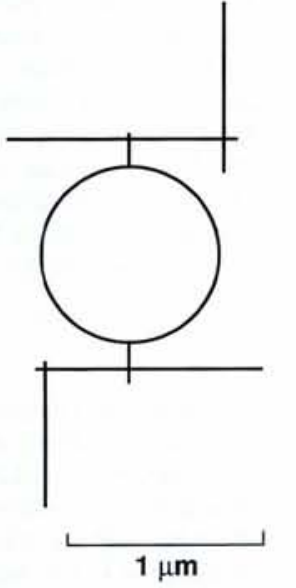

Fig. 5 - Mesoscopic effects in a normally conducting metallic loop: the interplay between periodic Bohm-Aharonov oscillations and random fluctuations. a, left) A drawing taken from a scanning electron micrograph of a $825 \mathrm{~nm}$ in diameter gold loop with drawn wires having thick ness $38 \mathrm{~nm}$ and width $49 \mathrm{~nm}$. b upper right) The dimensionless conductance of the loop over a narrow range of magnetic field in teslas $T$. (c, lower right) The Fourier transform of the data after subtraction of the average resistance contains peaks near $1 / \Delta H=0,130 / T$, and $260 / T$. The peaks correspond to random fluctuations, the hc/e oscillations, and the hc/2e oscillations, respectively. [Webb et al., 1985]. 
harmonics of an alternating current electric field in centro-symmetric crystals and nonlinear current/voltage characteristics in metals [Altshuler \& Khmelnitskii, 1985; Khmelnitskii \& Larkin, 1986]. Another example is the thermoelectric effect in metals which is known to be very small due to an approximate symmetry between electrons and holes near the Fermi level. In mesoscopic samples, this symmetry is violated as well, and mesoscopic fluctuations of the thermoelectric effect can be larger than an ensemble averaging effect [Anisovich, Altshuler, Aronov \& Zyuzin, 1987].

\section{Non-identical ensembles}

It is unnecessary to study an ensemble of macroscopically identical samples in order to observe the random fluctuations. Any method which changes the interference pattern will give the same result as changing the positions of impurities. The following examples illustrate this principle.

a) The Aharonov-Bohm mechanism may be used to change the interference pattern [Altshuler \& Khmelnitskii, 1985; Imry, 1985; Stone, 1985]. The random tangle of paths through the sample will generate different interference patterns depending on the flux threaded among paths. The characteristic magnetic field which changes the interference patterns $B_{\mathrm{c}}$ is given by $B_{\mathrm{c}} \approx \rho_{0} / S$ where $S$ is the area of the sample's crosssection perpendicular to $B$. The changes in magnetic field $B$ on $B_{c}$ lead to $g$ changing by an amount of the order of $e^{2 / \hbar}$ (Fig. 4).

b) The magnetoresistance of a single one-dimensional loop has Aharonov-Bohm oscillations with the period $\phi_{0}=h c / e$ (not the $h c / 2 e$ for weak localization). An actual loop is formed of a wire of finite width so the magnetoresistance involves a rather complicated interplay among the random fluctuations and periodic oscillations (Fig. 5); the amplitude of oscillations is of the order $e^{2 / h}$.

c) The interference pattern in a sample depends on the positions of impurities and it is very sensitive to the repositioning of a single impurity [Altshuler \& Spivak, 1985]. If $m$ impurities move in an uncorrelated fashion over the distance of the order of the electron wavelength, the variance in conductance is of the order $\left(e^{2 / \hbar}\right)^{2}$ (if $m>$ $\left.g \hbar / e^{2}\right)$. Such a high sensitivity of the universal conductance fluctuations to small variations in the impurity potential offers a new way to study the motion of heavy particles in metals.

\section{Non-locality}

The main feature of all the properties of disordered metals is their non-local character caused by quantum coherence in a random potential. Consequently, information about initial conditions is transferred by an electron for a large distance $L_{\varphi}=\sqrt{D \tau_{\varphi}}$ (or $L_{T}=\sqrt{\hbar D / T}$ where $T$ is the temperature) which exceeds the mean free path $I$. This feature - absolutely essential for understanding the physics of metallic spin glasses - leads immediately to an exchange interaction between two localized spins in metals (the so-called RKKI interaction), which has the characteristic radius of the order of $L_{0}$ or $L_{T}$, but not / [Bulaevskii, 1986; Spivak \& Zuyzin, 1986].
Non-locality leads to the non-local dependence of the electric current on the electric field [Aronov, Spivak \& Zyuzin, 1986] in disordered conductors. Local currents are larger than the average current along the average electric field, and they generate a magnetic moment on the sample. There is one interesting consequence of the non-local nature of the electron paths: this is where an experiment includes the effects of a ring which is outside the classical current path. For example, if a loop dangles outside the current path, hcle oscillations can manifest themselves on measuring the resistance, and this has been demonstrated experimentally [Webb et al., 1987]. So we see that electrons are non-local objects and this has remarkable and measurable consequences.

\section{ACKNOWLEDGEMENTS}

In the course of writing this appreciation I had many useful discussions with my colleagues, all of whom I wish to thank very sincerely, especially Y.B. Levinson who made some important comments. I would also like to thank Mrs. H. Fischer for help in preparing the text.

\section{REVIEWS}

Aronov A.G. \& Sharvin Yu.V., Rev. Mod. Phys. 59 (1987) 755.

Altshuler B.L. \& Aronov A.G., in ElectronElectron Interactions in Disordered Systems, Eds.: A.L. Efros \& M. Pollak (Elsevier, 1985) 1. Lee P.A. \& Ramakvrishnan T.V., Rev. Mod. Phys. 57 (1985) 1039.

Chakravary S. \& Schmid A., Phys. Rev. 140 (1986) 193.

Washburn W., in Mesoscopic Phenomena in Solids, Eds.: B.L. Altshuler, P.A. Lee \& R.A. Webb (Elsevier, 1991) 37.

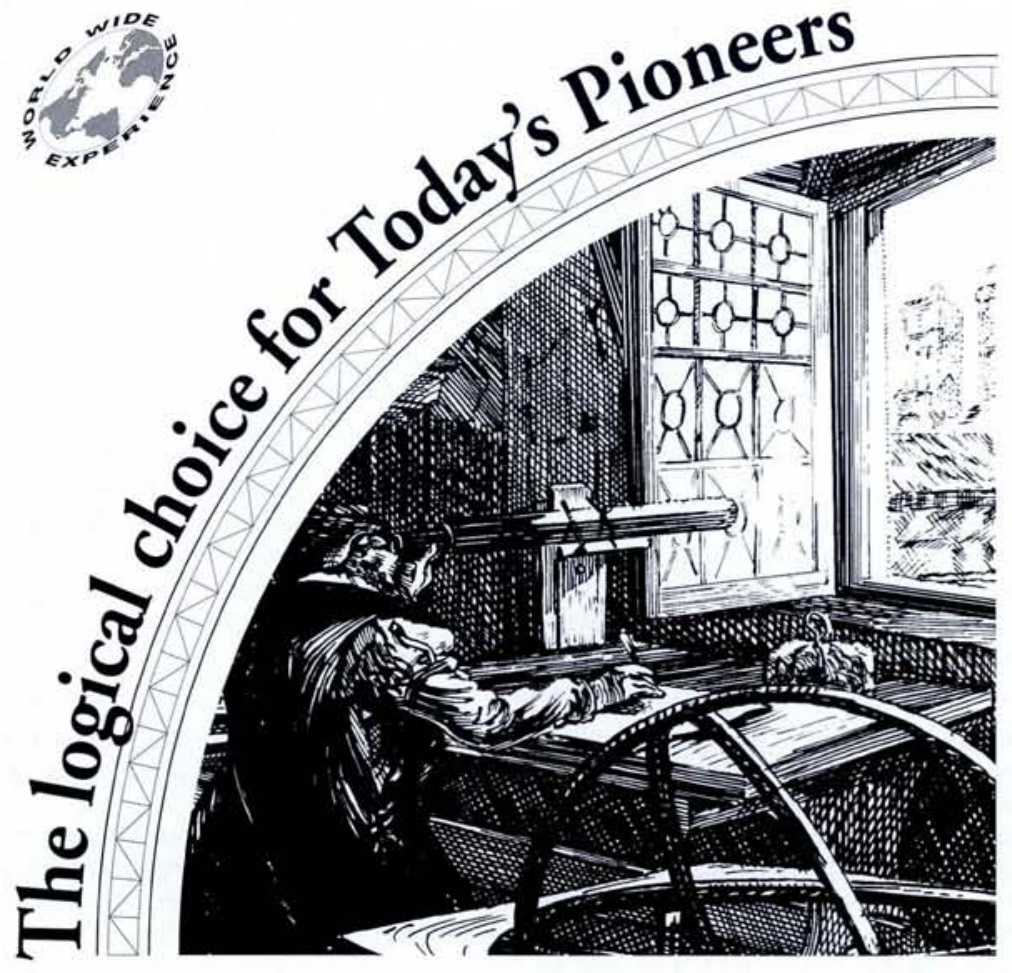

Just as for the pioneering natural philosophers of yesteryear, the key to new discoveries is so often an advance in instrumentation. The ability to see new phenomena depends on having state-of-the-art equipment incorporating the latest designs.

Tomorrows research equipment must be innovative, reliable and practical to use. The staff at Cryogenic Ltd are dedicated to providing just this - designed and built for your needs, making it possible to carry your research that bit further.

Add this to excellent personal before and after sales service from a team of dedicated experienced professionals and you have the blue print of Cryogenic Ltd.

To find out why many of today's pioneers choose Cryogenic Ltd, call us for information on our product range of low temperature equipment, susceptometers, magnets and SQUIDS, and make The Better Choice... logically.

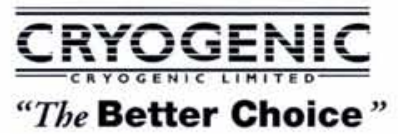

Cryogenic Ltd, Unit 30, Acton Park Industrial Estate, The Vale, London W3 7QE. Tel: (+44) 817436049 Fax (+44) 817495315 